\title{
Transduodenal endoscopy
}

\author{
SEAN F. O'BEIRN
}

From the Department of Surgery, University College, Galway, and the Regional Hospital, Galway, Eire

The technique to be described is presented as a useful addition to the repertoire of the surgeon who finds nothing abnormal in an abdomen during an operation for haematemesis (case 3), and also where at laparotomy an expected lesion of the stomach is not found, and a full examination of the mucosa is desired (case 10).

The approach was prompted by the easy and atraumatic fashion in which the second part of the duodenum can be brought level with the anterior abdominal wall during the first stage of the operation of pancreato-duodenal resection. Incidentally it is emphasized that the successful execution of this step supplies the key to a uniformly satisfactory examination.

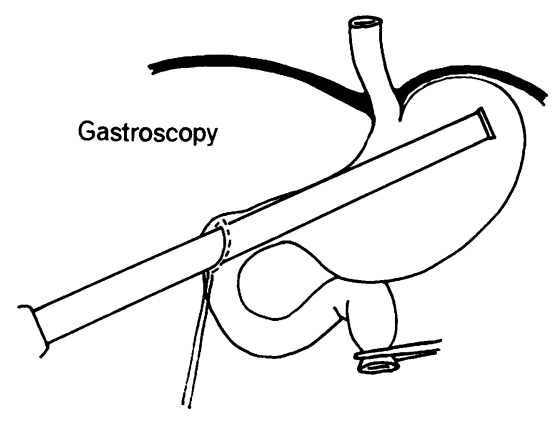

FIG. 1 .

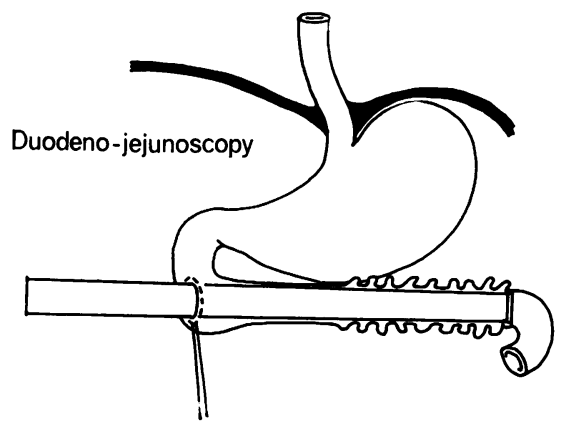

FIG. 3.
Although originally planned to allow complete visualization and biopsy of the stomach alone, the technique also provides an approach for inspection and biopsy of the lower oesophagus, the whole of the duodenum, including the papilla of Vater, and a variable amount of the jejunum.

\section{TECHNIQUE}

1 Mobilize the duodenum as for the first stage of pancreato-duodenal resection, and isolate the second part at the level of the anterior abdominal wall with packs.

2 Through a $\frac{1}{2}$ in. incision surrounded by a pursestring suture at the middle of the convex border of the second part of the duodenum introduce a standard

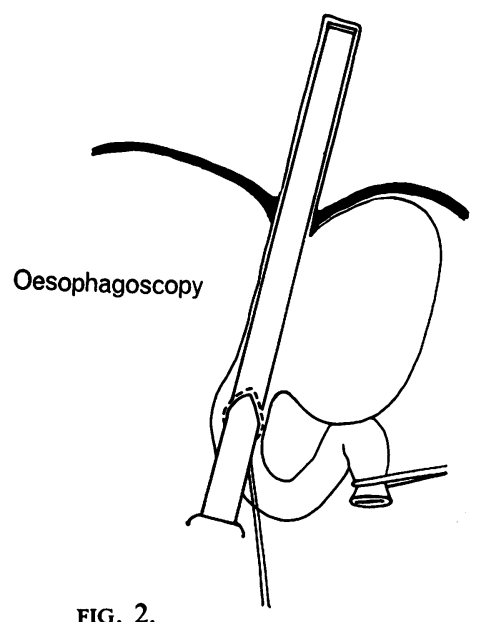

FIG. 2 .

FIG. 1. Duodenogastroscopy. Examine first and second parts of duodenum and negotiate pylorus using bellows. Examine stomach. Clamps on jejunum optional.

FIG. 2. Oesophagoscopy. Negotiate cardia-oesophageal junction using bellows and examine lower oesophagus. Clamps on jejunum optional.

FIG. 3. Duodenojejunoscopy. Distal duodenum and upper jejunum are examined if necessary before withdrawing endoscope and drawing purse-string tight. 
sterilized sigmoidoscope and pull the purse string tight.

3 From now on, the bellows of the sigmoidoscope are used to inflate the bowel and a standard punch biopsy forceps, such as Yeoman's, to collect biopsy material where indicated. Clamping the proximal jejunum helps to inflate the stomach and duodenum. It has not been found necessary to pack the oesophagus.

4 The instrument is now guided through the upper duodenum (which is examined in passing) and then through the pylorus. This passage is usually quite easy but occasionally may need help from a hand outside the bowel and very rarely must the obturator be reintroduced into the sigmoidoscope. The stomach wall is examined by spiral viewing, with the occasional inflation to flatten out folds (Fig. 1). A hand in front of the stomach helps to bring the anterior wall into view. Excess secretion is sucked out to ensure a clear view. If it is thought necessary, the oesophagus is entered through the oesophagogastric junction (Fig. 2). A Ryle's tube in the stomach acts as a very useful guide to this opening, which otherwise may be a little difficult to locate from inside the stomach. Before the instrument is withdrawn from the duodenum, the sphincter of Oddi, the distal duodenum, and the proximal jejunum may be viewed and biopsied if thought necessary (Fig. 3).

5 The duodenal opening is closed with $2 / 0$ catgut in two layers or by any other acceptable technique.

\section{CASE REPORTS}

The investigation has been carried out on 10 occasions. In six cases the oesophagus was also examined, and in five the jejunum. In all cases an excellent view was obtained and biopsy specimens were collected at will. The following reports illustrate the main indications.

CASE 3 A man aged 64 years presented with gross and continuing melaena, negative barium meal examination, and negative laparotomy findings. At transduodenal endoscopy a bleeding ulcer was located on the posterior wall of the duodenum and partial gastrectomy carried out.

CASE 10 A man aged 60 years had previously had a partial gastrectomy for adenocarcinoma of the antrum. A barium meal examination was highly suggestive of recurrence at the anastomosis site and gastric cytology was positive. At laparotomy a thickening was found at one area of the previous anastomosis site. At transduodenal endoscopy no recurrence was found and biopsies taken at various points along the anastomosis line, at the fundus, and at the greater curvature were all negative.

\section{COMPLICATIONS}

A slight, self-correcting bleed occurred in one patient, and a more severe one in another due to over-vigorous use of the biopsy forceps. One unit of blood was sufficient to correct this. There was no mortality or other morbidity.

\section{CONTRAINDICATIONS}

It is necessary to stress that unless the second part of the duodenum can be brought level with the anterior abdominal wall, the full examination will probably fail. Furthermore, since the full examination can add half an hour to the operating time and subject the patient to slightly increased trauma, its use in older patients, and particularly those with cardiovascular or constitutional defects, must be approached with caution. In very stout people (and those with deformities) the use of this examination could well be too difficult to justify except under very special circumstances. Finally, it must be mentioned that the ease with which adequate biopsy material can be obtained introduces its own hazard of excessive postoperative bleeding. Care and common sense are needed in taking biopsy specimens.

\section{SUMMARY}

A transduodenal approach for endoscopic examination and biopsy of the stomach, lower oesophagus, duodenum, and upper jejunum has been evolved. The indications, contraindications, and complications are discussed, and a brief report on two typical cases is included.

I am indebted to Dr T. Warner for the diagrams. 